\section{Inverted internal limiting membrane flap technique as a useful procedure for macular hole- associated retinal detachment in highly myopic eyes}

H Sasaki, A Shiono, J Kogo, R Yomoda,

Y Munemasa, M Syoda, H Otake, H Kurihara,

Y Kitaoka and H Takagi

\begin{abstract}
Purpose To determine whether the inverted internal limiting membrane (ILM) flap technique contributes to high reattachment and closure rates in patients with macular hole-associated retinal detachment (MHRD). Patients and methods In all, 15 eyes of $\mathbf{1 5}$ patients with MHRD undergoing 25-gauge pars plana vitrectomy with the inverted ILM flap technique or ILM peeling. The patients were divided into the inverted ILM flap technique group (6 eyes) and ILM peeling group (9 eyes). The logarithm of minimal angle of resolution best-corrected visual acuity (BCVA) and retinal attachment and macular hole closure rates were compared between the two groups before and after surgery.
\end{abstract}

Results No significant differences were found in the pre- and postoperative BCVA at 1 and 3 months after surgery in either group (inverted ILM flap technique group, preoperatively $1.04 \pm 0.55,1$ month $0.95 \pm 0.30,3$ months $0.83 \pm 0.22$; ILM peeling group, preoperatively $1.00 \pm 0.44,1$ month $1.05 \pm 0.38,3$ months $1.06 \pm 0.49 ; P>0.05$, respectively). The postoperative BCVA at 6 months after surgery was significantly better in the inverted ILM flap technique group than in the ILM peeling group (inverted ILM flap technique group, $0.62 \pm 0.35$; ILM peeling group, $1.02 \pm 0.41$, $P=0.045)$. The improvement in BCVA was significantly better in the inverted ILM flap technique group than in the ILM peeling group (inverted ILM flap technique group, $-0.41 \pm 0.29$; ILM peeling group, $0.02 \pm 0.36$; $P=0.021$ ). The primary macular hole closure rates were $\mathbf{1 0 0} \%$ in the inverted ILM flap technique group and $55.5 \%$ in the ILM peeling group. The primary reattachment rates were $100 \%$ in the inverted ILM flap technique group and $55.5 \%$ in the ILM peeling group. The primary macular hole closure and reattachment rates were not significantly different in both groups ( $P=0.056$, respectively).

Conclusion The inverted ILM flap technique is a useful procedure for MHRD in highly myopic eyes.

Eye (2017) 31, 545-550; doi:10.1038/eye.2016.263; published online 2 December 2016

\section{Introduction}

Macular hole-associated retinal detachment (MHRD) occurs most commonly in myopic eyes and is an intractable form of retinal detachment. ${ }^{1-3}$ Several approaches have been attempted such as macular buckling, scleral shortening, pars plana vitrectomy (PPV), and combinations thereof. ${ }^{4-6}$ The macular buckling technique was the standard surgical treatment for MHRD before the introduction of PPV in 1982. ${ }^{2,7-9}$ The retinal attachment rate after macular buckling and scleral shortening ranged from 93.3 to $100 \%$, although the macular hole closure rates after these procedures were unknown. ${ }^{4}$ Since then, PPV has gained popularity with several additional procedures such intraocular gas or silicon oil tamponade, ${ }^{10-12}$ laser photocoagulation of the macular hole rim, ${ }^{12}$ and internal limiting membrane (ILM) peeling. ${ }^{13-15}$ The retinal reattachment rates after these initial surgeries ranged from 42.8 to $92.3 \%{ }^{13,16-19}$ Previous reports showed that the macular hole closure
Department of Ophthalmology, St Marianna University School of Medicine, Kawasaki, Japan

Correspondence: J Kogo, Department of Ophthalmology, St Marianna University School of Medicine, 2-16-1 Sugao, Miyamae-ku, Kawasaki 216-8511, Japan Tel: +81 44977 8111; Fax: +81 449767435 E-mail: kogo@

marianna-u.ac.jp 
rate using optical coherence tomography (OCT) after PPV ranged from 10 to $91 \% .{ }^{13,15}$ These anatomic success rates were variable and not always greater than in cases of nonmacular hole-related rhegmatogenous retinal detachment and idiopathic macular hole. Therefore, the basic surgical management of MHRD has not been clarified.

Recently, Michalewska et $a l^{20}$ have reported the efficacy of the ILM flap technique for large macular holes. Kuriyama and Michalewska et al ${ }^{21,22}$ also found that the inverted ILM flap technique contributed to a high macular hole closure rate in highly myopic eyes. We therefore hypothesized that the inverted ILM flap technique would be an effective treatment for MHRD. The aim of this study was to determine whether the technique could contribute to high reattachment and closure rates in patients with MHRD.

\section{Patients and methods}

This study protocol was approved by St Marianna University Hospital and adhered to the tenets of the Declaration of Helsinki. We reviewed the medical records of consecutive patients who had MHRD and were treated by PPV with the inverted ILM flap technique or ILM peeling at St Marianna University Hospital, Kurihara Eye Hospital, and Otake Eye Clinic between October 2009 and October 2013. Between October 2009 and April 2013, the consecutive patients underwent PPV with ILM peeling. From May 2013, the inverted ILM flap technique was performed in consecutive patients. Before and after surgery, all patients underwent a through ocular examination, including determination of axial length and visual acuity, and biomicroscopy of the posterior segment using a slit lamp with a $90 \mathrm{D}$ lens. The preoperative diagnosis and postoperative anatomic status of MHRD were examined using OCT. High myopia was defined as an axial length $>27 \mathrm{~mm}$. Staphyloma was defined as ectasia in the posterior pole detected in OCT. The bestcorrected visual acuity (BCVA) was measured using the Landolt $\mathrm{C}$ acuity test and converted to a logarithm of minimal angle of resolution for statistical analysis. The follow-up period was at least 6 months after the final surgery. Exclusion criteria were a history of trauma, choroidal neovascularization, and the presence of a peripheral retinal break or proliferative vitreoretinopathy before the initial surgery.

A standard 25-gauge PPV and phacoemulsification with intraocular lens implantation was performed in eyes that had cataracts, and vitrectomy alone was performed in pseudophakic eyes and eyes without cataracts. Triamcinolone acetonide was used intraoperatively to facilitate visualization of the vitreous and posterior hyaloids in all eyes. Subsequently, the inverted ILM flap technique was performed in six eyes. ILM peeling and removal were performed in nine eyes. For better visibility, we stained the ILM with a solution of brilliant blue G (BBG), as described by Enaida et $a l^{23}$ The inverted ILM flap technique was basically performed according to the method of Michalewska et $a l^{20}$ To stabilize the ILM flap, we placed a Viscoat ophthalmic viscosurgical device (sodium chondroitin sulfate-sodium hyaluronate; Alcon Laboratories, Inc., Fort Worth, TX, USA) on the inverted ILM. Fluid-air exchange up to the staphyloma margin or slightly above the upper margin of the detached retina was performed if the retinal detachment was localized within the arcade. When the retinal detachment extended to or beyond the equator, drainage of subretinal fluid was performed through the retinal hole intentionally created outside the macular hole in patients undergoing the inverted ILM flap technique and through the macular hole in patients undergoing ILM peeling. In all patients, the air was then replaced with nonexpansive gas, either perfluoropropane or sulfur hexafluoride. Each patient was asked to maintain a facedown position postoperatively for at least 5 days.

All procedures were performed by a single surgeon (HT). To evaluate the efficacy of the inverted ILM flap technique for MHRD, the patients were divided into two groups based on the surgical procedure: the inverted ILM flap technique group (group 1) and ILM peeling group (group 2). The BCVA and retinal attachment and macular hole closure rates were compared between the two groups before and after surgery.

Statistical analyses were performed using StatView software (Abacus Concepts, Inc., Berkeley, CA, USA, 1992). The Wilcoxon rank-sum test was used to compare pre- and postoperative BCVA. The $\chi^{2}$-test was used to compare retinal attachment and macular hole closure rates. The comparison of BCVA in the two groups was analyzed in the Mann-Whitney $U$-test. A P-value of $<0.05$ was considered to represent a statistically significant difference.

\section{Results}

Patient characteristics are shown in Table 1. In all, 12 women (80\%) and 3 men (20\%) were included in this study. The patient ages at the initial surgery ranged from 46 to 82 (mean $69.6 \pm 11.2$ ) years. All patients had staphyloma. Table 2 shows a comparison of preoperative patient demographic data. Group 1 included 6 eyes of 6 patients in whom vitrectomy combined with the inverted ILM flap technique was performed, and group 2 included 9 eyes of 9 patients in whom vitrectomy combined with ILM peeling for MHRD was performed (Figure 1). The duration of symptoms, age, axial length, and preoperative BCVA did not differ significantly between them $(P>0.05$, respectively). Secondary surgery was performed in 3 eyes 
Table 1 Patient characteristics

\begin{tabular}{|c|c|c|c|c|c|c|c|c|c|c|}
\hline $\begin{array}{l}\text { Patient } \\
\text { no. }\end{array}$ & Age & Gender & Eye & $\begin{array}{c}\text { Duration of } \\
\text { symptoms (weeks) }\end{array}$ & $\begin{array}{l}\text { Preoperative } \\
\text { lens status }\end{array}$ & $\begin{array}{c}\text { Axial } \\
\text { length }(\mu \mathrm{m})\end{array}$ & $\begin{array}{l}\text { Operative } \\
\text { procedure }\end{array}$ & $\begin{array}{l}\text { Inverted } \\
\text { ILM flap }\end{array}$ & Tamponade & $\begin{array}{c}\text { Preoperative BCVA } \\
\text { (decimal) }\end{array}$ \\
\hline 1 & 78 & F & $\mathrm{R}$ & 28 & Phakic & 27.14 & PPV, PEA, IOL & - & $\mathrm{C}_{3} \mathrm{~F}_{8}$ & 0.09 \\
\hline 2 & 71 & $\mathrm{~F}$ & $\mathrm{R}$ & 16 & IOL & 27.24 & PPV & + & $\mathrm{SF}_{6}$ & 0.02 \\
\hline 3 & 77 & $\mathrm{~F}$ & $\mathrm{R}$ & 24 & IOL & 28.49 & PPV & - & $\mathrm{SF}_{6}$ & 0.02 \\
\hline 4 & 50 & $\mathrm{~F}$ & $\mathrm{~L}$ & 8 & IOL & 31.22 & PPV & - & $\mathrm{C}_{3} \mathrm{~F}_{8}$ & 0.5 \\
\hline 5 & 74 & $\mathrm{~F}$ & $\mathrm{~L}$ & 2 & Phakic & 28.72 & PPV, PEA, IOL & - & $\mathrm{C}_{3} \mathrm{~F}_{8}$ & 0.04 \\
\hline 6 & 77 & $\mathrm{~F}$ & $\mathrm{R}$ & 4 & Phakic & 31.61 & PPV, PEA, IOL & + & $\mathrm{C}_{3} \mathrm{~F}_{8}$ & 0.03 \\
\hline 7 & 82 & $\mathrm{~F}$ & $\mathrm{R}$ & 4 & IOL & 28.34 & PPV & + & $\mathrm{SF}_{6}$ & 0.3 \\
\hline 8 & 64 & M & $\mathrm{R}$ & 4 & Phakic & 29.74 & PPV & + & $\mathrm{SF}_{6}$ & 0.5 \\
\hline 9 & 68 & M & $\mathrm{R}$ & 2 & IOL & 31.14 & PPV & - & $\mathrm{C}_{3} \mathrm{~F}_{8}$ & 0.05 \\
\hline 10 & 74 & $\mathrm{~F}$ & $\mathrm{~L}$ & 16 & IOL & 29.14 & PPV & - & $\mathrm{C}_{3} \mathrm{~F}_{8}$ & 0.09 \\
\hline 11 & 73 & $\mathrm{~F}$ & $\mathrm{R}$ & 48 & IOL & 31.4 & PPV & - & $\mathrm{C}_{3} \mathrm{~F}_{8}$ & 0.3 \\
\hline 12 & 46 & $\mathrm{~F}$ & $\mathrm{R}$ & 6 & IOL & 30.06 & PPV & - & $\mathrm{C}_{3} \mathrm{~F}_{8}$ & 0.2 \\
\hline 13 & 79 & $\mathrm{~F}$ & $\mathrm{~L}$ & 1 & IOL & 34.37 & PPV & + & $\mathrm{SF}_{6}$ & 0.06 \\
\hline 14 & 54 & M & $\mathrm{R}$ & 4 & Phakic & 33.63 & PPV, PEA, IOL & - & $\mathrm{SF}_{6}$ & 0.1 \\
\hline 15 & 77 & $\mathrm{~F}$ & $\mathrm{~L}$ & 12 & IOL & 31.56 & PPV & + & $\mathrm{C}_{3} \mathrm{~F}_{8}$ & 0.1 \\
\hline
\end{tabular}

Abbreviations: $\mathrm{BCVA}$, best-corrected visual acuity; $\mathrm{C}_{3} \mathrm{~F}_{8}$, perfluoropropane; $\mathrm{F}$, female; ILM, internal limiting membrane; IOL, intraocular lens; $\mathrm{M}$, male; $\mathrm{PPV}$, pars plana vitrectomy; $\mathrm{PEA}$, phacoemulsification; $\mathrm{SF}_{6}$, sulfur hexafluoride.

Table 2 Comparison of preoperative study participant data (group 1=inverted ILM flap technique; group 2=ILM peeling)

\begin{tabular}{|c|c|c|c|}
\hline Characteristics & Group 1 & Group 2 & P-value \\
\hline No. of patients & 6 & 9 & \\
\hline Age (years; mean $\pm S D)$ & $75.0 \pm 6.4$ & $66.0 \pm 12.5$ & 0.125 \\
\hline Gender (male/female) & $1 / 5$ & $2 / 7$ & \\
\hline Duration of symptoms (week; mean \pm SD) & $6.8 \pm 5.8$ & $11.3 \pm 9.5$ & 0.376 \\
\hline \multicolumn{4}{|l|}{ Preoperative lens status } \\
\hline Phakic & 2 & 3 & \\
\hline Pseudophakic & 4 & 6 & \\
\hline Preoperative RD status (within the archades/beyond the archades) & $1 / 5$ & $3 / 6$ & \\
\hline Axial length (mm; mean $\pm S D)$ & $30.47 \pm 2.57$ & $30.10 \pm 1.95$ & 0.637 \\
\hline \multicolumn{4}{|l|}{ Tamponade } \\
\hline $\mathrm{C}_{3} \mathrm{~F}_{8}$ & 1 & 6 & \\
\hline $\mathrm{SF}_{6}$ & 5 & & \\
\hline Preoperative BCVA $(\log M A R ;$ mean $\pm S D)$ & $1.04 \pm 0.55$ & $1.00 \pm 0.44$ & 0.906 \\
\hline
\end{tabular}

Abbreviations: $\mathrm{BCVA}$, best-corrected visual acuity; $\mathrm{C}_{3} \mathrm{~F}_{8}$, perfluoropropane; ILM, internal limiting membrane; logMAR, logarithm of minimum angle of resolution; $\mathrm{RD}$, retinal detachment; $\mathrm{SF}_{6}$, sulfur hexafluoride.

in group 2, and 1 patient refused secondary surgery (Supplementary Figure 1).

Postoperative ophthalmic data are shown in Table 3. No significant differences were found in the postoperative BCVA at 1 and 3 months after surgery in both groups $(P>0.05$, respectively). The postoperative BCVA at 6 months after surgery was significantly better in group 1 than in group $2(P=0.045)$. The improvement of BCVA was significantly better in group 1 than in group 2 $(P=0.021)$. The primary macular hole closure rates were $100 \%$ in group 1 and $55.5 \%$ in group $2(P=0.056)$. The final macular hole closure rates were $88.8 \%$ in group 2 . There was no significant difference in both groups
$(P=0.398)$. The primary reattachment rates were $100 \%$ in group 1 and $55.5 \%$ in group $2(P=0.056)$. The final reattachment rates were $100 \%$ in group 1 and $88.8 \%$ in group $2(P=0.398)$.

\section{Discussion}

In the current study, we demonstrated that the primary macular hole closure and reattachment rates after PPV with the inverted ILM flap technique reached 100\%, which were higher anatomic success rates compared with patients who underwent ILM peeling alone. In anatomical success rates, the statistical analysis differences were not 

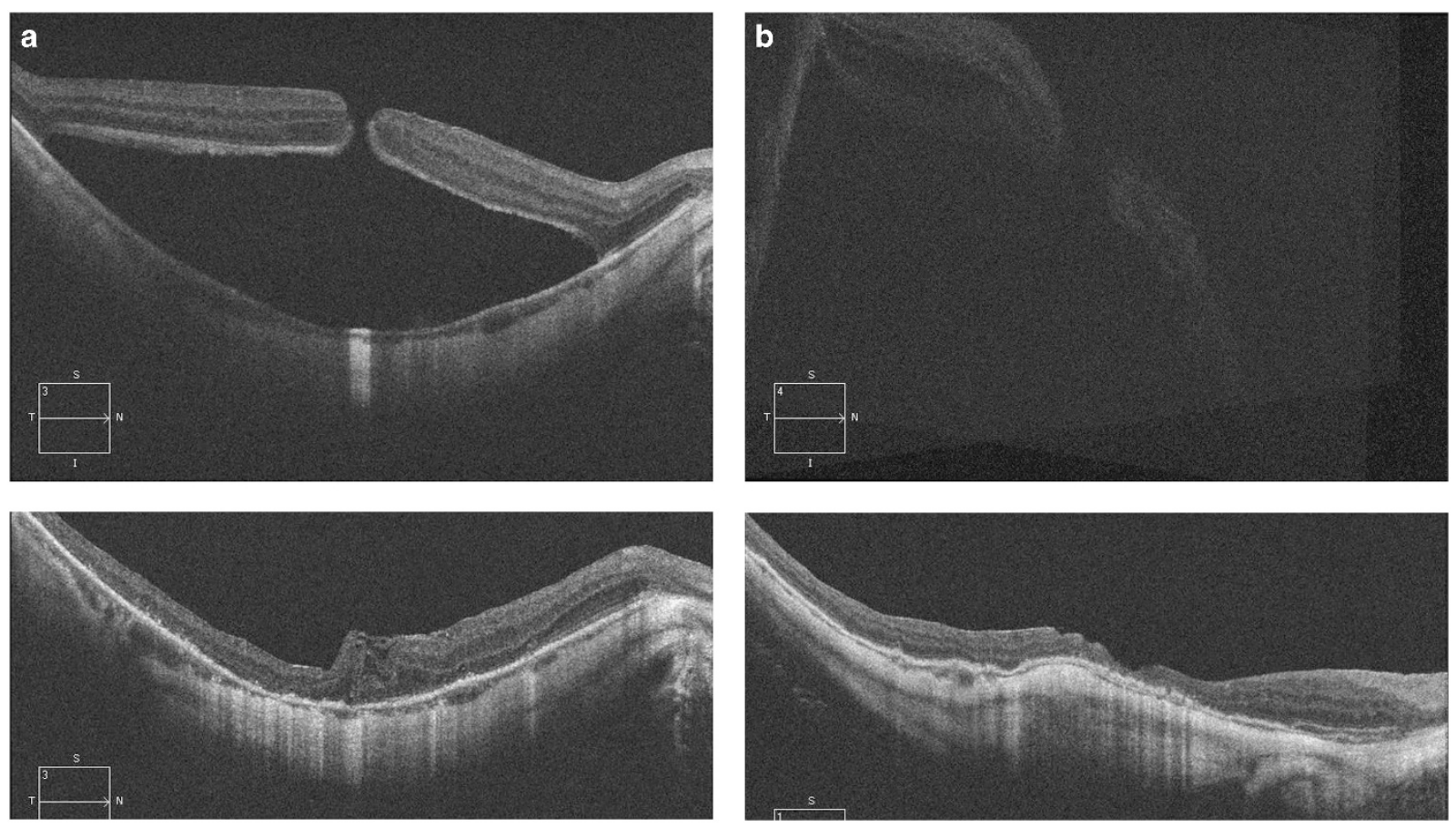

Figure 1 (a) A 64-year-old man presented with MHRD. OCT demonstrated a macular hole and retinal detachment within the arcade (top left). The initial BCVA was 20/40. Using primary 25-gauge PPV with the inverted ILM flap technique, the ILM was inverted. Six months after surgery, the retina was reattached and the macular hole closed (bottom left). Postoperative BCVA was 20/40. (b) A 71-yearold woman presented with MHRD. OCT demonstrated a macular hole and retinal detachment beyond the arcade (top right). The initial BCVA was 4/200. Using primary 25-gauge PPV with the inverted ILM flap technique, the ILM was inverted. One month after surgery, the retina was reattached and the macular hole closed (bottom right). Postoperative BCVA improved to 16/200.

Table 3 Comparison of postoperative study participant data (group $1=$ inverted ILM flap technique; group $2=$ ILM peeling)

\begin{tabular}{lccc}
\hline Characteristics & Group 1 & Group 2 & P-value \\
\hline Postoperative BCVA (logMAR; mean \pm SD) & & & \\
$\quad 1$ month & $0.95 \pm 0.30$ & $1.05 \pm 0.38$ & 0.68 \\
3 months & $0.83 \pm 0.22$ & $1.06 \pm 0.49$ & 0.238 \\
$\quad 6$ months & $0.62 \pm 0.35$ & $1.02 \pm 0.41$ & 0.045 \\
Improvement in BCVA & $-0.41 \pm 0.29$ & $0.02 \pm 0.36$ & 0.021 \\
Primary MH closure rate & $100 \%(6 / 6)$ & $55.5 \%(5 / 9)$ & 0.056 \\
Final MH closure rate & $100 \%(6 / 6)$ & $88.8 \%(8 / 9)$ & 0.398 \\
Primary retinal reattachment rate & $100 \%(6 / 6)$ & $55.5 \%(5 / 9)$ & 0.056 \\
Final reattachment rate & $100 \%(6 / 6)$ & $88.8 \%(8 / 9)$ & 0.398 \\
\hline
\end{tabular}

Abbreviations: BCVA, best-corrected visual acuity; ILM, internal limiting membrane; logMAR, logarithm of minimum angle of resolution MH, macular hole.

determined, but patients who underwent the inverted ILM flap technique tended to achieve high anatomical success rates. Several groups reported the efficacy of ILM peeling as the initial procedure for MHRD. ${ }^{13,14,24}$ In this procedure, the macular hole closure and reattachment rates were $10-91 \%$ and $70-92.3 \%$, respectively. Our results showed higher anatomic success rates after PPV with the inverted ILM flap technique compared with previous reports.
ILM peeling allows total removal of the cortical vitreous and its components, contributing to complete relaxation of the retina. However, the retinal tension cannot be eliminated completely by ILM peeling, as some of the components, that is, vascular traction and posterior staphyloma, cannot be eliminated. ILM peeling alone may not compensate for macular hole closure in high myopia. Michalewska et al hypothesized that the inverted ILM flap technique stimulates proliferation of glial cells that fill the 
macular hole. In histopathologic findings, the ILM serves as a scaffold for gliosis. ${ }^{25}$ One possibility is that proliferating glial cells may fill the macular hole and compensate for retinal shortening.

In terms of postoperative visual prognosis, the postoperative BCVA at 6 months after surgery and improvement of BCVA were significantly better in group 1 than in group 2. These results suggest that the proliferation of glial cells produces an environment conducive to the repositioning of photoreceptors in direct proximity to the fovea.

In a comparison of the inverted ILM flap technique and ILM peeling alone, Chen et al ${ }^{26}$ reported that 23-gauge PPV with the inverted ILM flap technique contributed to better anatomical success. However, they found that there were no statistically significant differences in postoperative BCVA and improvement of BCVA between the inverted ILM flap technique and ILM peeling. One possibility to explain this is that their surgical procedures differed from ours. The difference may also have been due to the dyes used. Chen et $\mathrm{l}^{26}$ used indocyanine green (ICG) as the adjuvant, and many researchers ${ }^{27-29}$ reported the possible negative effects of ICG on visual outcome. Previously, other groups, ${ }^{27,28}$ including ours, ${ }^{30}$ reported that ICG staining may negatively affect the recovery of macular morphology and visual acuity after macular hole surgery. Baba et $a l^{31}$ reported that the postoperative BCVA was better in eyes stained with BBG than in those stained with ICG, and the restoration of the ellipsoid zone was faster in eyes stained with BBG. In this procedure, the stained ILM flap comes into direct contact with the retinal pigment epithelium and remains stained over a long period. Therefore, we used BBG for the inverted ILM flap technique and ILM peeling alone.

In terms of anatomic success, our study showed that no significant differences were found in the anatomical success rates in both groups, but patients who underwent the inverted ILM flap technique achieved high anatomical success. An important problem is the possibility of the ILM flap moving away from the hole opening in the inverted ILM flap technique. Recently, Lai et $a l^{32}$ have introduced the novel technique of using autologous blood clots to stabilize and seal ILM flaps. That technique has shown high anatomic success rates and only requires patients with MHRD to remain in a facedown position for a short time postoperatively. Lai et al ${ }^{32}$ found that blood clots seal the holes after application and that the blood components and growth factors also facilitate healing. However, our study showed excellent anatomic success rates without the use of autologous blood clots. Further investigations are required to determine whether autologous blood clot application is effective in combination with the inverted ILM flap technique.
This study had certain limitations. The number of eyes was relatively small, the study was retrospective, and the follow-up period was short. Consequently, further studies are needed to confirm these results. In conclusion, the inverted ILM flap technique is a useful procedure for MHRD in highly myopic eyes and may become the standard treatment in the future.

\author{
Summary \\ What was known before \\ - The basic surgical management of MHRD is not obvious. \\ What this study adds \\ - The inverted ILM flap technique is a useful procedure \\ for MHRD.
}

\section{Conflict of interest}

The authors declare no conflict of interest.

\section{References}

1 Cheung BT, Lai TY, Yuen CY, Lai WW, Tsang CW, Lam DS. Results of high-density silicone oil as a tamponade agent in macular hole retinal detachment in patients with high myopia. Br J Ophthalmol 2007; 91: 719-721.

2 Siam AL. Macular hole with central retinal detachment in high myopia with posterior staphyloma. Br J Ophthalmol 1969; 53: 62-63.

3 Akiba J, Konno S, Yoshida A. Retinal detachment associated with a macular hole in severely myopic eyes. Am J Ophthalmol 1999; 128: 654-655.

4 Ando F, Ohba N, Touura K, Hirose H. Anatomical and visual outcomes after episcleral macular buckling compared with those after pars plana vitrectomy for retinal detachment caused by macular hole in highly myopic eyes. Retina 2007; 27: $37-44$.

5 Meng L, Wei W, Li Y, Han X, Shi X, Yang M. Treatment of retinal detachment secondary to macular hole in highly myopic eyes. Retina 2014; 34: 470-476.

6 Alkabes M, Bures-Jelstrup A, Salinas C. Macular buckling for previously untreated and recurrent retinal detachment due to high myopic macular hole: a 12-month comparative study. Grafes Arch Clin Exp Ophthalmol 2014; 252: 571-581.

7 Margherio RR, Schepens CL. Macular breaks. II. Management. Am J Ophthalmol 1972; 74: 233-240.

8 Feman SS, Helper RS, Straatsma BR. Rhegmatogenous retinal detachment due to macular hole. Management with cryotherapy and a Y-shaped sling. Arch Ophthalmol 1974; 91: 371-372.

9 Gonvers M, Machemer R. A new approach to treating retinal detachment with macular hole. Am J Ophthalmol 1982; 94: 468-472.

10 Miyake Y. A simplified method of treating retinal detachment with macular hole. Arch Ophthalmol 1986; 104: 1234-1236. 
11 Blankenship GW, Ibanez-Langlois S. Treatment of myopic macular hole and detachment. Intravitreal gas exchange. Ophthalmology 1987; 94: 333-336.

12 Wolfensberger TJ, Gonvers M. Long-term follow-up of retinal detachment due to macular hole in myopic eyes treated by temporary silicone oil tamponade and laser photocoagulation. Ophthalmology 1999; 106: 1786-1791.

13 Kadonosono K, Yazawa F, Itoh N, Uchio E, Nakamura S, Akura J et al. Treatment of retinal detachment resulting from myopic macular hole with internal limiting membrane removal. Am J Ophthalmol 2001; 131: 203-207.

14 Ichibe M, Yoshizawa T, Murakami K, Ohta M, Oya Y, Yamamoto $S$ et al. Surgical management of retinal detachment associated with myopic macular hole: anatomic and functional status of the macula. Am J Ophthalmol 2003; 136: 277-284.

15 Ikuno Y, Sayanagi K, Oshima T, Gomi F, Kusaka S, Kamei M et al. Optical coherence tomography findings of macular holes and retinal detachment after vitrectomy in highly myopic eyes. Am J Ophthalmol 2003; 136: 477-481.

16 Kuriyama S, Matsumura M, Harada T, Ishigooka H, Ogino N. Surgical techniques and reattachment rates in retinal detachment due to macular hole. Arch Ophthalmol 1990; 108: 1559-1561.

17 Lu L, Li Y, Cai S, Yang J. Vitreous surgery in highly myopic retinal detachment resulting from a macular hole. Clin Exp Ophthalmol 2002; 30: 261-265.

18 Seiki C, Kusaka S, Sakagami K, Ohashi Y. Reopening of macular hole in highly myopic eyes with retinal detachment. Retina 1997; 17: 2-6.

19 Ripandelli G, Coppe AM, Fedeli R, Parisi V, D'Amico DJ, Stirpe M. Evaluation of primary surgical procedures for retinal detachment with macular hole in highly myopic eyes: a comparison of vitrectomy versus posterior episcleral buckling surgery. Ophthalmology 2001; 108: 2258-2264.

20 Michalewska Z, Michalewska J, Adelman RA, Nawrocki J. Inverted internal limiting membrane flap technique for large macular holes. Ophthalmology 2010; 117: 2018-2025.

21 Kuriyama S, Hayashi H, Jingami Y, Kuramoto N, Akita J, Matsumoto $\mathrm{M}$ et al. Efficacy of internal limiting membrane flap technique for the treatment of macular hole in high myopia. Am J Ophthalmol 2013; 156: 125-131.

22 Michalewska Z, Michalewska J, Dulczewska-Cichecha K, Nawrocki J. Inverted internal limiting membrane flap technique for surgical repair of myopic macular holes. Retina 2014; 34: 664-669.

23 Enaida H, Hisatomi T, Hata Y, Ueno A, Goto Y, Yamada T et al. Brilliant blue $\mathrm{G}$ selectively stains the internal limiting membrane/brilliant blue G-assisted membrane peeling. Retina 2006; 26: 631-636.

24 Uemoto R, Yamamoto S, Tsukahara I, Takeuchi S. Efficacy of internal limiting membrane removal for retinal detachments resulting from a myopic macular hole. Retina 2004; 24: 560-566.

25 Madreperla SA, Geiger GL, Funata M, de la Cruz Z, Green WR. Clinicopathologic correlation of a macular hole treated by cortical vitreous peeling and gas tamponade. Ophthalmology 1994; 101: 682-686.

26 Chen SN, Yang CM. Inverted internal limiting membrane insertion for macular hole-associated retinal detachment in high myopia. Am J Ophthalmol 2016; 162: 99-106.

27 Engelbrecht NE, Freeman J, Sternberg Jr P, Aaberg Sr TM, Aaberg Jr TM, Martin DF et al. Retinal pigment epithelial changes after macular hole surgery with indocyanine greenassisted internal limiting membrane peeling. Am J Ophthalmol 2002; 133: 89-94.

28 Sakamoto T, Itaya K, Noda Y, Ishibashi T. Retinal pigment epithelial changes after indocyanine green-assisted vitrectomy. Retina 2002; 22: 794-796.

29 Kusaka S, Oshita T, Ohji M, Tano Y. Reduction of the toxic effect of indocyanine green on retinal pigment epithelium during macular hole surgery. Retina 2003; 23 : 733-734.

30 Shiono A, Kogo J, Klose G et al. Effect of indocyanine green staining on the recovery of visual acuity and macular morphology after macular hole surgery. Ophthalmologica 2013; 230: 138-143.

31 Baba T, Hagiwara A, Sato E, Arai M, Oshitari T, Yamamoto S. Comparison of vitrectomy with brilliant blue $\mathrm{G}$ or indocyanine green on retinal microstructure and function of eyes with macular hole. Ophthalmology 2012; 119: 2609-2615.

32 Lai CC, Chen YP, Wang NK, Chuang LH, Liu L, Chen KJ et al. Vitrectomy with internal limiting membrane repositioning and autologous blood for macular hole retinal detachment in highly myopic eyes. Ophthalmology 2015; 122: 1889-1898.

This work is licensed under a Creative NoDerivs 4.0 International License. The images or other third party material in this article are included in the article's Creative Commons license, unless indicated otherwise in the credit line; if the material is not included under the Creative Commons license, users will need to obtain permission from the license holder to reproduce the material. To view a copy of this license, visit http://creativecommons.org/licenses/by-nc-nd/4.0/

(C) The Author(s) 2017

Supplementary Information accompanies this paper on Eye website (http://www.nature.com/eye) 